Revue d'histoire de l'Amérique française

REVUE D.HISTOIRE DE L'AMÉRIQUE FRANÇAISE

\title{
La Conquête de 1760 et le problème de la transition au capitalisme
}

\section{Claude Couture}

Volume 39, numéro 3, hiver 1986

URI : https://id.erudit.org/iderudit/304372ar

DOI : https://doi.org/10.7202/304372ar

Aller au sommaire du numéro

Éditeur(s)

Institut d'histoire de l'Amérique française

ISSN

0035-2357 (imprimé)

1492-1383 (numérique)

Découvrir la revue

Citer cet article

Couture, C. (1986). La Conquête de 1760 et le problème de la transition au capitalisme. Revue d'histoire de l'Amérique française, 39(3), 369-389.

https://doi.org/10.7202/304372ar d'utilisation que vous pouvez consulter en ligne.

https://apropos.erudit.org/fr/usagers/politique-dutilisation/ 


\title{
LA CONQUETE DE 1760 ET LE PROBLEME DE LA TRANSITION AU CAPITALISME ${ }^{1}$
}

\author{
CLAUDE COUTURE \\ Département d' histoire \\ Université de Montréal
}

Après avoir analysé, dans des articles parus en 1972 et 1974, l'historiographie de la Nouvelle-France entre 1900 et $1960^{2}$, l'historien Jean Blain a écrit: «La risposte qui contient des promesses d'avenir vient des sociologues, des politicologues et autres représentants des sciences sociales...» ${ }^{3}$.

En ce qui concerne les politicologues, certains auteurs se sont effectivement chargés, notamment au début des années 80, d'assurer cette «riposte». Ainsi, en 1981, Gérald Bernier proposait aux lecteurs de la Revue d'histoire de l'Amérique française et de la Revue canadienne de science politique une nouvelle interprétation de la Conquête de 1760 fondée sur l'idée d'une «rupture structurelle» de la socio-économie de l'ancienne Nouvelle-France provoquée par l'avènement d'une métropole «plus capitaliste» ${ }^{4}$. Au printemps de l'année suivante, $\mathrm{M}$. Ta Van, qui avait participé aux premiers travaux de Gérald Bernier sur le sujet, publiait dans les Cahiers d' histoire de l'Université de Montréal un article reprenant essentiellement la même thèse $\mathrm{e}^{5}$. Puis, en septembre 1982, Gérald Bernier, cette fois en compagnie de Daniel Salée, étonnait ses lecteurs de la $R H A F$ en dressant un portrait révisé de la bourgeoisie

\footnotetext{
I Plusieurs personnes ont patiemment lu les premières versions de ce texte. Je tiens cependant à remercier tout particulièrement les professeurs Pierre Tousignant, Louis Michel, Pierre Trépanier et Jacques Rouillard, du département d'histoire de l'Université de Montréal, Jean-Pierre Wallot, maintenant archiviste fédéral, Yves Bourdon, étudiant au doctorat en histoire à l'Université de Montréal, Alain Noël, étudiant au doctorat en science politique à la Graduate School of International Studies de Denver et Dominique Jean, étudiante au doctorat en histoire, actuellement en scolarité à l'Université de Boston.

2 Jean Blain, «Économie et société en Nouvelle-France: le cheminement historiographique dans la première moitié du 20e siècle», Revue d'histoire de l'Amérique française, 26,1 (juin 1972): 3-33, et, «Économie et société en Nouvelle-France: l'historiographie des années 50-60», RHAF, 28,2 (septembre 1974): 163-176.

3 Jean Blain, op. cit., (septembre 1974): 185-186.

4 Gérald Bernier, «Sur quelques effets de la rupture structurelle engendrée par la Conquête au Québec: 1760-1854», RHAF, 35,1 (juin 1981): 69-95; «La structure de classes québécoises au $19 \mathrm{e}$ siècle et le problème de l'articulation des modes de production", Revue canadienne de science politique, 14,3 (septembre 1981): 487-518 (surtout p. 494).

5 Ta Van, «La Nouvelle-France et la Conquête: passage du féodalisme au capitalisme», Cahiers d'histoire, 2,2 (printemps 1982): 3-25.
} 
britannique qui s'installa dans la vallée du Saint-Laurent après la Conquête ${ }^{6}$.

Dans cet article, Gérald Bernier et Daniel Salée ont voulu démontrer que la bourgeoisie britannique au Canada, entre 1763 et 1846, était en apparence capitaliste mais au fond féodale, parce qu'elle oeuvrait surtout au niveau de la reproduction de la «sphère d'échange» (capitalisme marchand) et non au niveau de la «sphère de production» (capitalisme industriel). Un an plus tôt, Gérald Bernier avait pourtant écrit, comme nous le verrons plus en détail, qu'en raison de l'intervention d'une métropole «plus proche» de la phase industrielle du capitalisme, les institutions «féodales» héritées du régime français furent insérées dans une logique d'accumulation du capital menant à une accélération de la transition du féodalisme au capitalisme. Or, comme les marchands britanniques qui se sont installés après 1763 et qui ont acheté des seigneuries furent responsables de l'implantation de cette «dynamique capitaliste» exportée par la nouvelle métropole dans l'ancienne Nouvelle-France, on en déduit que ces marchands-seigneurs étaient au fond capitalistes bien qu'en apparence féodaux.

Cette «apparente» contradiction entre les deux articles est d'autant plus surprenante que ces analyses, à première vue séduisantes, se voulaient plus globales, plus rigoureuses et mieux articulées théoriquement que celles de l'historiographie, qu'elle soit nationaliste ou, au contraire, «creightonienne». Même Fernand Ouellet, peu inquiet du fait d'être l'une des principales cibles de Gérald Bernier, a manifesté un intérêt pour ces travaux lorsqu'il a écrit dans un bilan historiographique récent: "Gérald Bernier et Daniel Salée sont en voie de mettre au point une autre version marxiste plus attentive à la question nationale des transformations de la société québécoise au cours du siècle qui suit la Conquête. ${ }^{7}$ Mais à y regarder de plus près, ces contributions sont peutêtre empreintes de tares plus graves encore que celles décrites par Jean Blain au sujet d'une historiographie trop centrée sur un «concept d'histoire nationaliste». Comme Gérald Bernier et Ta Van soutiennent principalement l'idée d'une «rupture structurelle» engendrée par la Conquête et l'avènement d'une métropole "plus capitaliste», c'est sur ce point que nous concentrerons notre critique.

Plus précisément, nous verrons que ces auteurs pèchent par une ignorance, lourde de conséquences, de la complexité de la révolution industrielle en Angleterre; qu'une abondante littérature en histoire économique a depuis belle lurette infirmé les conclusions de Marx au sujet

\footnotetext{
6 Gérald Bernier et Daniel Salée, «Appropriation foncière et bourgeoisie marchande: éléments pour une analyse de l'économie marchande du Bas-Canada avant 1846", RHAF, 36,2 (septembre 1982): 163-194.

Fernand Ouellet, «Les classes dominantes au Québec, 1760-1840. Bilan historiographique», RHAF, 38,2 (automne 1984): 242.
} 
de la «disparition de la yeomanry» anglaise après 1750 et la formation d'une main-d'oeuvre «nue et libre», phénomènes que d'aucuns jugent essentiels à la transition au capitalisme industriel; qu'il est chimérique, par conséquent, d'essayer de retrouver pour le Bas-Canada de la fin du $18 \mathrm{e}$ siècle et du premier tiers du $19 \mathrm{e}$, certains phénomènes «universels», résultant des «lois de l'histoire» régissant le passage du féodalisme au capitalisme, alors que lesdits phénomènes ne se sont même pas produits dans le pays «modèle», en l'occurrence l'Angleterre; qu'en dernière instance, il semble que l'on ait projeté sur l'Angleterre des années 1760 les bouleversements économiques des années 1820-1850, de sorte que toute la démonstration pourrait bien reposer sur un anachronisme.

Mais d'abord, en quoi consiste exactement, selon Gérald Bernier et Ta Van, la «rupture structurelle» engendrée par la Conquête?

\section{1 - LA RUPTURE STRUCTURELLE ENGENDRÉE PAR LA CONQUETE SELON GERALD BERNIER}

$\mathrm{Au}$ contraire des historiens «libéraux» et sociologues marxistes qualifiés de «continuistes» parce qu'ils ont écrit que la Conquête n'a pas fondamentalement modifié la socio-économie de l'ancienne Nouvelle-France ${ }^{8}$, Gérald Bernier entend démontrer que les événements des années 1759-1763 ont «amorcé une rupture structurelle» ${ }^{9}$.

Cette rupture structurelle résulterait de l'insertion «brutale», au sein d'une société dominée par le mode de production féodal, de structures reflétant «le capitalisme le plus avancé de l'époque» ${ }^{10}$. Globalement, ces changements structuraux auraient signifié «l'inversion de la matrice de dominance du procès d'articulation des modes de production rencontrés sous la Nouvelle-France ${ }^{11}$. En d'autres mots, avant la Conquête, dans l'articulation des deux modes de production, le féodalisme prédominait. Après 1760 , et sans que le renversement soit le résultat d'une impulsion interne, le mode de production capitaliste devint dominant à la suite du changement de métropole.

Tout repose en effet sur le fait que la France du milieu du 18e siècle serait féodale et moins capitaliste dans sa phase transitoire que la Grande-Bretagne ${ }^{12}$. Gérald Bernier attribue cette avance des Britanniques principalement au phénomène de la concentration des terres en Angleterre marqué par les «enclosures» et à la formation d'une «main-

\footnotetext{
${ }^{8}$ Gérald Bernier, «Sur quelques effets de la rupture structurelle engendrée par la Conquête au Québec: 1760-1854», op. cit., 69, note 1. Les auteurs visés sont Fernand Ouellet et Jean Hamelin, d'une part, Gilles Bourque, Anne Légaré et Nicole Frenette, d'autre part.

9 Ibid.

10 Ibid., 70

11 Ibid.

12 Ibid., 71, note 4.
} 
d'oeuvre nue et libre» provoquée par l'expropriation de la petite paysannerie ${ }^{13}$. Après la Conquête, l'Angleterre aurait ainsi «exporté» ses éléments structuraux dans sa nouvelle colonie ${ }^{14}$.

S'amorce alors pour la formation canadienne la phase de transition du féodalisme au capitalisme puisque sont déclenchés, dans la logique même du capitalisme anglais, le procès d'expropriation et de prolétarisation de la paysannerie et le renversement du procès d'articulation des modes de production avec l'instauration du MPC comme matrice dominante. (...) Car le renforcement du caractère féodal du régime seigneurial, durant le premier deux-tiers de siècle qui suit la Conquête, (...) n'est qu'apparent. Sa signification ne répond plus à la logique de la surexploitation féodale, mais bien à celle capitaliste de constituer, à plus ou moins long terme, une base d'accumulation du capital et une main-d'oeuvre nue et libre. ${ }^{15}$

Dans le cas de la nouvelle dynamique capitaliste et des tendances à l'accumulation accrue, les effets auraient été immédiats. Par contre, dans celui de la formation d'une masse de travailleurs «nus et libres», le phénomène se serait graduellement manifesté de 1760 à 1850 . Au coeur de ce processus, le rôle des marchands anglais a été, évidemment, essentiel. Bernier affirme que ces marchands auraient très rapidement adapté les seigneuries qu'ils ont achetées après la Conquête aux lois du marché parce qu'ils étaient «mieux rompus aux lois du capitalisme» ${ }^{16}$. Cependant, les activités de ces marchands-seigneurs seraient liées à la phase marchande (procès de circulation) du mode de production capitaliste. Et, consciemment, ces marchands-seigneurs auraient plutôt cherché à maintenir le statu quo, c'est-à-dire la dominance du procès de circulation (phase marchande) sur le procès de production (phase industrielle). Ce serait donc malgré eux qu'ils auraient contribué à accélérer l'avènement de la phase industrielle du mode de production capitaliste en accumulant des capitaux et en provoquant la formation d'une main-d'oeuvre libre.

Paradoxal fut également le rôle de la nouvelle métropole. En effet, si cette métropole a pu, en amont, fouetter la dynamique capitaliste après la Conquête, elle a exercé, en aval, une série de contraintes sur la colonie qui a prolongé la «phase marchande». Car pour la bourgeoisie métropolitaine, «la colonie existe d'abord en fonction de l'assouvissement de ses propres besoins, en l'occurrence pour s'approvisionner en matières premières et produits primaires et ouvrir une source de débouchés à ses produits manufacturés» ${ }^{17}$. Ainsi, la dimension coloniale a «médiatisé» le procès d'expulsion de la paysannerie par la bour-

\footnotetext{
13 Ibid.

14 Ibid.

5 Ibid., 76-77.

Ibid., 82

17 Ibid., 89.
} 
geoisie en freinant l'industrialisation. D'où «la mise en place des mécanismes conduisant à l'expulsion de la paysannerie avant même que ne soit développée la capacité d'absorption de ce surplus» ${ }^{18}$. D'où, conséquemment, l'émigration vers les Etats-Unis de Canadiens français et le fait que la première génération de prolétaires québécois se serait constituée outre-frontières. Ce n'est qu'après 1846-1851, au moment où l'Angleterre adopte le libre-échange, que les conditions réelles et complètes de développement du capitalisme industriel au Canada seront mises en place.

Nouvelle dynamique capitaliste, accumulation accrue et formation d'une main-d'oeuvre disponible provoquée par l'expropriation de la paysannerie. Voilà, aux yeux de Gérald Bernier, les éléments essentiels de la rupture structurelle engendrée par la Conquête et du renversement de la «matrice de dominance» en faveur du mode de production capitaliste, phénomènes qui seraient les manifestations concrètes des «lois de l'histoire» régissant le passage du féodalisme au capitalisme ou, au moment de la transition, de la phase marchande à la phase industrielle ${ }^{19}$. Voilà pourquoi, également, les institutions héritées du régime français ne sont restées «féodales» qu'en apparence seulement puisque, fondamentalement, leur fonction après la Conquête fut de préparer l'émergence du capitalisme de la phase industrielle sous l'instigation d'une bourgeoisie marchande britannique inconsciente, agissant, selon Gérald Bernier, dans la plus parfaite ignorance des «lois de l'histoire».

\section{2 - LA CONQUETE REVUE ET CORRIGÉE PAR TA VAN}

Pour sa part, Ta Van s'attaque à l'historiographie en général, qu'elle soit «néo-nationaliste» ou «libérale» ${ }^{20}$. Si la Conquête correspond à une rupture, ce n'est pas au sens d'un «traumatisme national», mais plutôt comme conséquence de l'insertion dans la dynamique d'une nouvelle métropole capitaliste. Par contre, la Conquête ne fut pas non plus un «progrès» parce que le développement de l'industrie dans la colonie est freiné par la nature impérialiste de la nouvelle métropole.

18 Ibid., 94.

19 Ibid., 89. Bernier a écrit: «En bref, l'orientation prise par l'agriculture sous l'impulsion de la bourgeoisie marchande porte les germes du propre dépassement de cette classe en ce qu'il mène, à plus ou moins long terme, à l'émergence du capitalisme de la phase industrielle. Telle n'est certes pas la volonté de la bourgeoisie marchande (...), mais tel est précisément le résultat, lorsque les diverses dispositions sont appréciées à la lumière des lois de l'histoire en ce qui concerne les étapes (le souligné est de nous) régissant le passage du féodalisme au capitalisme et, plus proprement, à l'intérieur du MPC lui-même, le passage de la phase marchande à la phase industrielle.»

${ }_{20}$ Ta Van, op. cit., 3. L'auteur a notamment écrit: «Longtemps les quelques escarmouches entre les classiques en ce qui concerne la féodalité de la Nouvelle-France et la signification de la Conquête britannique ont dominé la scène: nous les avons subies, et nous les supportons encore. Pourtant pour reprendre ces tropes, si elles ont beau émouvoir quelques vagues et dessiner des rides à la surface, ce ne sont que tempêtes au bassin des enfants.» 
La thèse, on le voit bien, est essentiellement la même que celle de Gérald Bernier. Ta Van ajoute cependant que si l'historiographie n'a pas su saisir la signification de la Conquête, c'est parce que les historiens n'ont pas compris «que les rapports féodaux ne se définissent pas (...) à partir de la grandeur ou de la quantité des redevances, mais du fait même de l'existence du surtravail extorqué» ${ }^{21}$. La Nouvelle-France était donc féodale, en raison, toujours selon Ta Van, de la prédominance des activités agricoles et de la production paysanne, sources du «surtravail extorqué» ${ }^{22}$. Le commerce des fourrures y occupait une place marginale et le mercantilisme faisait partie intégrante du féodalisme.

Tout aurait changé avec la Conquête. «Première transformation: elle a détaché le mercantilisme de la métropole française et avec altération essentielle l'intègre de force à l'Angleterre. Le capitalisme s'est rabattu sur le féodalisme» ${ }^{23}$. Ce «rabattement» aurait été rendu possible par le fait que l'Angleterre était le seul pays, au milieu du 18e siècle, où l'on retrouvait le capitalisme «sous sa forme la plus achevée». Comment expliquer cette avance? Par deux phénomènes: la séparation des paysans de leurs moyens de production et la «capitalisation» des rapports de production dans l'agriculture à la suite des fameux «enclosure acts»; l'émergence d'une fraction capitaliste foncière comme classe politique dominante à la suite des révolutions du 17e siècle (1642-1660 et 1688$)^{24}$.

En somme, la concentration de la fortune mercantile et foncière et la formation d'une main-d'oeuvre «nue et libre» au milieu du 18 e siècle, résultat de l'expropriation de la petite paysannerie, seraient, selon Ta Van, les éléments clefs de la transition du féodalisme au capitalisme. Une transition qui se serait d'abord effectuée en Angleterre pour se généraliser universellement par la suite puisque, ne l'oublions pas, il s'agit-là de lois de l'histoire. Le Bas-Canada n'a évidemment pas échappé à ces supposées «lois». Et Ta Van de conclure, après avoir ainsi «désintriqué» les mécanismes profonds des transformations engendrées par la Conquête: «La littérature historiographique n'est au fond qu'une parole qui dit $n$ 'importe quoi et ne se tait jamais.» ${ }^{25}$

\section{LA RÉVOLUTION INDUSTRIELLE, L'ANGLETERRE ET LA TRANSITION AU CAPITALISME}

\section{$A$ - La «disparition» de la «yeomanry» anglaise}

Les assertions des auteurs cités reposent essentiellement sur l'hypothèse que l'Angleterre du 18 e siècle a été «le premier pays à réunir

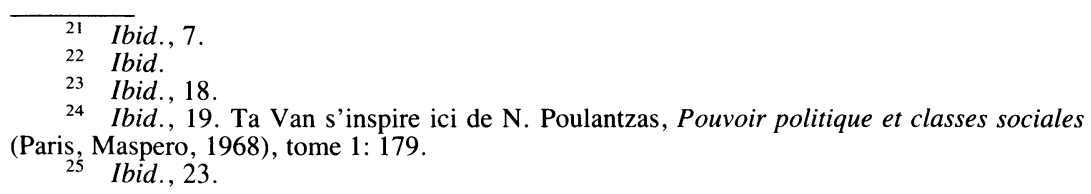


les conditions qui favorisent l'apparition du capitalisme de la phase industrielle ${ }^{26}$. Et l'élément le plus déterminant, sur lequel insistent Gérald Bernier et Ta Van, fut que «l'Angleterre de 1760 amorçait les temps forts du procès d'expropriation de la paysannerie - facteur essentiel pour la constitution d'un prolétariat» ${ }^{27}$.

La thèse est classique. Elle a déjà été formulée par Karl Marx puis reprise par de nombreux auteurs, notamment Paul Mantoux, et même dans un ouvrage récent de Michel Beaud ${ }^{28}$. Dans les campagnes anglaises, particulièrement celles du sud, les initiatives des grands propriétaires nobles menèrent à la disparition du système de l'openfield. Phénomène séculaire, les enclosures des terres communales s'intensifièrent tout au long du 18e siècle. De 1730 à 1820 , elles furent même sanctionnées par plus de 5000 «Acts of Enclosures» ${ }^{29} .75000$ acres de terres communales furent ainsi enclos de 1727 à 1760; 478000 de 1760 à 1792 et 1000000 de 1792 à $1815^{30}$. Quelques contemporains ont été très impressionnés par les conséquences économiques et sociales de ce mouvement, surtout dans certains comtés. S'inspirant de leurs témoignages, Marx vit dans la concentration des terres et la disparition des communaux la possibilité de la formation d'une surpopulation rurale prête à gagner les villes manufacturières naissantes et à servir de maind'oeuvre non-qualifiée, en somme de prolétariat. Ainsi, les squatters et les cottagers, naguère simples occupants des communaux, disparurent des campagnes et gagnèrent les villes. Même chose en ce qui concerne les petits et moyens propriétaires indépendants - les yeomen qui auraient dû quitter, eux aussi, les campagnes anglaises. En se référant notamment à une lettre datée de 1795 d'un gentleman du Suffolk, dans le sud-est de l'Angleterre, à Sir T. C. Banbury, «avocat fanatique du système des grandes fermes», Marx a même écrit au sujet de ces yeomen: «Vers 1750, la yeomanry avait disparu.» 31

Mais est-ce si sûr? Depuis les années 1930, dans le cadre d'enquêtes en histoire régionale, plusieurs historiens économistes anglais ont étudié les mouvements migratoires de la population anglaise entre 1750 et 1815, à partir d'évaluations de la population des comtés au $18 \mathrm{e}$ siècle et des données du début du $19 \mathrm{e}$ siècle sur le système d'assistance à domicile (Speenhamland system of poor relief). Les conclusions aux-

\footnotetext{
26 Gérald Bernier, «La rupture structurelle...», op. cit., 71, note 4.

27 Gérald Bernier, «La structure de classes québécoises...», op. cit., 494, note 4.

28 Paul Mantoux, La Révolution industrielle au 18e siècle (Paris, Génin, 1959) (première édition en 1928; version corrigée d'une thèse présentée en 1906), 577 pages. Michel Beaud, Histoire du capitalisme: 1500-1980 (Paris, Seuil, 1981), 37-43.

29 J.-P. Rioux, La révolution industrielle (Paris, Seuil, 1971), 31.

30 Phyllis Deane, The First Industrial Revolution (Cambridge, Cambridge University Press, 1965), 42; (second edition, 1978). pitre 27.
} 
quelles ils arrivent sont fort différentes de celles de Marx. Dès 1937, il a été démontré que pour le comté de Suffolk, entre 1797 et 1815 , le nombre des petits propriétaires avait augmenté par rapport à la période d'avant 1750, donc avant l'intensification des enclosures, et qu'en aucun temps il y eut de migration vers le nord «industriel» ${ }^{32}$. Même phénomène en ce qui concerne les 119 villages du comté de Nottinghamshire dans le centre-est de l'Angleterre ${ }^{33}$. De 1780-1786 à 1832, la population rurale de ce comté a augmenté à un rythme légèrement moins rapide que celle des villes en voie d'industrialisation et n'a jamais reculé. Fait encore plus intéressant, la population des villages «enclos» a progressé plus rapidement que celle des villages d'openfield. Le scénario se vérifie dans le comté de Lindsey, partie de l'ancien comté du Lincolnshire ${ }^{34}$.

Après plus de trente ans de recherche en histoire régionale de l'Angleterre, on en arrive généralement à la conclusion suivante: les yeomen, loin de disparaître en 1750, ont en fait été progressivement plus nombreux pendant la deuxième moitié du $18 \mathrm{e}$ siècle et la population rurale anglaise n'a cessé d'augmenter jusqu'au milieu du $19 \mathrm{e}$ siècle ${ }^{35}$. Comment cela a-t-il été possible?

Il faut d'abord prendre en considération la chronologie économique du $18 \mathrm{e}$ siècle. La période 1720-1760 fut marquée par une dépression des prix agricoles, notamment ceux du blé, qui «lamina» les profits des producteurs anglais, petits ou grands, et les rendit peu enclins à

32 P. Lavrosky, «Parliamentary Enclosures in the County of Suffolk (1797-1814)», Economic History Review, 7 (1937), cité dans J. D. Chambers, «Enclosure and Labour Supply in the Industrial Revolution», Economic History Review, 5,3 (1952-1954): 325.

33 J. D. Chambers, op . cit., 323.

34 J. D. Chambers, «Enclosure and the Small Landowner», Economic History Réview, 10,2 (1940): 118-127.

35 Plusieurs auteurs ont critiqué ces études. E. P. Thompson a écrit dans The Making of the English Working Class (Harmondsworth, Penguin Books, 1968), 238-239: «(...) the social violence of enclosure consisted precisely in the drastic, total imposition upon the village of capitalist property-definitions. (...) Enclosure (...) was of profound social consequence, because it illuminates, both backwards and forwards, the destruction of the traditional elements in English peasant society.» Barrington Moore dans Les origines sociales de la dictature et de la démocratie (Paris, Maspero, 1969), 29, a exprimé le même point de vue: «Les historiens d'il y a un siècle pensaient que l'enclosure du 18e siècle avait été le principal expédient de la toute-puissante aristocratie terrienne pour en finir avec la paysannerie indépendante. Par la suite, on a lentement et patiemment ruiné cette opinion. Seuls peut-être quelques marxistes la soutiendraient encore aujourd'hui. Elle pèche évidemment dans le détail et parfois dans ses grandes lignes. On avait pourtant bien vu ce qu'on oublie aujourd'hui; les enclosures portent un coup fatal à la structure de la société paysanne anglaise telle qu'elle s'exprime dans le village traditionnel.» Que le village traditionnel et la culture populaire fondée sur l'openfield aient été détruits par les enclosures, que la société paysanne traditionnelle ait été bousculée par les méthodes «capitalistes», on l'admet volontiers. Mais là n'est pas la question qui nous préoccupe. Marx et plusieurs autres après lui ont bel et bien écrit que la yeomanry avait disparu vers 1750 et que la paysannerie dépossédée avait envahi les villes manufacturières naissantes de la deuxième moitié du $18 \mathrm{e}$ siècle. Or il n'en a rien été et continuer à l'affirmer relève de la fabulation ou de l'ignorance. Pour une critique détaillée et impitoyable des erreurs de Marx sur la Révolution agricole en Angleterre, voir Kins Collins, «Marx on the English Agricultural Revolution: Theory and Evidence», History and Theory, 6,3 (1967): 351381 . 
poursuivre la vague des innovations agricoles amorcée au $17 \mathrm{e}$ siècle ${ }^{36}$. Par contre, la période 1760-1815 en fut une de hausse des prix agricoles stimulés, entre autres, par un gonflement du marché interne dû à une augmentation sans précédent de la population anglaise. Alors que le nombre des habitants de l'Angleterre et du pays de Galles avait été relativement stable entre 1720 et 1760, il passa d'environ 6 millions en 1760 à 12 millions en $1811^{37}$. Dans un premier temps, ce doublement de la population en un demi-siècle fut attribuable à un recul des surmortalités cycliques causées par les épidémies et les crises de subsistance. Une population mieux nourrie, de meilleures conditions climatiques et hygiéniques, une «immunisation naturelle» de la population après plusieurs générations ravagées par la peste et la variole, auraient brisé le rythme macabre des crises cycliques de surmortalité. Puis, dans un deuxième temps, aiguillonné par une économie en expansion qui offrait de nouvelles possibilités d'emplois, se produisit un abaissement de l'âge au mariage provoquant une hausse déterminante du taux de natalité à partir de 1780 .

Révolution agricole et révolution démographique sont donc inextricablement liées. L'élargissement de l'offre d'emplois à la fin du $18 \mathrm{e}$ siècle, facteur essentiel à l'abaissement de l'âge au mariage, a résulté de la progression des innovations agricoles, elle-même inconcevable sans l'accroissement du marché interne et la hausse des prix agricoles après $1760^{38}$. Voulant justement profiter de la montée de ces prix, surtout ceux du blé, les propriétaires anglais se lancèrent dans la culture intensive. Celle-ci était caractérisée par des rotations accélérées, l'utilisation de plantes fourragères, la réduction de la jachère et l'extension des emblavures sur les terres communales ${ }^{39}$. La progression des enclosures apparaît alors non pas comme la victoire définitive de l'aris-

36 Louis Bergeron, «La «révolution agricole» en Angleterre», in Pierre Léon, Histoire économique et sociale du monde: 1730-1840 (Paris, Armand Colin, 1978), tome 3: 228.

37 W. A. Cole et Phyllis Deane, British Economic Growth: 1688-1959 (Cambridge, Cambridge University Press, 1962), 6-8.

38 E. A. Wrigley et R. S. Schofield, The Population History of England: 1541-1871 (Londres, Edward Arnold, 1981), 421-435. Ce phénomène de l'abaissement de l'âge au mariage à la fin du $18 \mathrm{e}$ siècle a été à l'origine d'un débat entre les partisans d'une vision «optimiste» de la révolution industrielle et les «pessimistes». En fait, il est certain que l'abaissement de l'âge au mariage à partir des années 1775-1780 est lié à une plus grande possibilité d'emplois générés par la révolution agricole et l'industrie manufacturière naissante. Mais cette plus grande possibilité d'emplois ne s'est pas traduite par une hausse des revenus suffisante pour combler l'augmentation du coût de la vie caractéristique de cette période. Aujourd'hui, on s'entend généralement sur une diminution du salaire réel entre 1760 et 1820 et une dégradation des conditions de vie des masses anglaises au cours de ces années, le fardeau des guerres entre 1793 et 1815 étant particulièrement lourd à porter. Cela dit, il ne faut pas confondre cet appauvrissement avec une disparition de la yeomanry qui ne s'est jamais produite entre 1760 et 1815 , ni avec un exode rural qui n'a pu se réaliser au moment où la révolution agricole retenait dans les campagnes une main-d'oeuvre abondante. Pour une mise à jour de cette question, voir Peter H. Lindert et Jeffrey Williamson, «English Workers' Living Standards During the Industrial Revolution», The Economic History Review, 36,2 (February 1983): 1-25.

Louis Bergeron, «La «révolution agricole» en Angleterre», op. cit., 227. 
tocratie sur la petite paysannerie, mais plutôt comme le triomphe de l'individualisme agraire... chez les grands comme chez les petits propriétaires. Voilà pourquoi, selon Phyllis Deane, il y eut en Angleterre, entre 1780 et 1815 , une augmentation, vérifiée par toutes les études en histoire régionale, du nombre de petits propriétaires exploitants:

(...) regional research for period after 1780 suggests that enclosure by Acts of Parliament produced an increase in all grades of occupying owners. Under the Enclosure Acts, many common-right cottagers received compensation for their rights which permitted them for the first time to buy small plots of land. ${ }^{40}$

Lorsque la conjoncture changera après 1815 , les petits propriétaires, incapables de soutenir la dépression des prix, disparaîtront peu à peu des campagnes ${ }^{41}$. Mais là encore, ce ne sera pas avant le deuxième tiers du $19 \mathrm{e}$ siècle que l'on assistera à un exode rural que Marx saura étudier avec plus de justesse ${ }^{42}$.

Par ailleurs, il n'y a pas lieu de penser que les laissés pour compte des enclosures, qui n'ont pu s'acheter un bout de terre, à savoir les cottagers (qui ne possèdent que leur maison), les squatters (occupants illégaux des terres communales) et les tenants at will (qui n'ont qu'une garantie verbale d'occupation des communaux), aient davantage pris le chemin des villes entre 1750 et 1800 . Le recours massif aux nouvelles techniques agricoles à partir de 1760 et les travaux d'enclosure comme tels ont provoqué dans les campagnes anglaises un écart entre l'offre d'emplois et la demande. Même l'augmentation spectaculaire de la population n'a pu y suffire, créant ainsi un problème de pénurie relative de main-d'oeuvre pendant la seconde moitié du $18 \mathrm{e}$ siècle jusqu'en 1815. «La nouvelle agriculture était labour-intensive» ${ }^{43}$ et des «bras» supplémentaires étaient requis pour une foule de travaux: «L'aménagement et l'entretien des clôtures, haies et fossés; le soin d'un bétail plus nombreux; le travail plus intensif d'un sol allégé de jachères et chargé de nouvelles cultures; la clôture des zones de forêts, de landes ou de marais.» Enfin, «le développement agricole a stimulé celui d'un petit commerce et d'un artisanat ruraux» ${ }^{44}$. Tous ces travaux ont retenu dans les campagnes une population nombreuse qui n'a cessé d'augmenter jusqu'au milieu du 19e siècle ${ }^{45}$.

En somme, si les enclosures ont fait d'une masse d'anciens utilisateurs des terres communales des ouvriers agricoles, elles n'ont eu

\footnotetext{
$40 \quad$ Phyllis Deane, The First Industrial Revolution, 44.

41 François Bédarida, L'Angleterre triomphante: 1833-1914 (Paris, Hatier, 1974), 27.

42 Karl Marx, op. cit., chapitre 25, 468-479, 492-525.

43 François Crouzet, «Agriculture et révolution industrielle. Quelques réflexions», Cahiers d'histoire, Lyon, 12 (1967): 72.

44 Louis Bergeron, «La «révolution agricole» en Angleterre», op. cit., 230.

45 Lawrence White, «Enclosures and Population Movements in England», Explorations in Entrepreneurial History, 6 (1968-69): 176-186.
} 
aucun rôle direct dans la formation d'une main-d'oeuvre industrielle. Dans les premières manufactures «modernes», c'est-à-dire les filatures de l'industrie cotonnière de la fin du $18 \mathrm{e}$ siècle et du début du $19 \mathrm{e}$, le recrutement s'est généralement fait sur place à partir des surplus démographiques et des premiers contingents d'immigrants irlandais ${ }^{46}$. Le véritable transfert de main-d'oeuvre vers les villes commencera avec «les difficultés économiques dues à la dépression des prix après 1815 , s'amplifiant considérablement avec la nouvelle Poor Law de 1834, (...) l'abolition des Corn Laws en $1846 »{ }^{47}$ et la fin des travaux d'enclosure. Plusieurs facteurs ont donc provoqué ce mouvement au milieu du $19 \mathrm{e}$ siècle et non les seules enclosures.

Or nous avons vu dans les démonstrations de Gérald Bernier et Ta Van que la Conquête de 1760 avait engendré une «rupture structurelle» de l'économie de l'ancienne Nouvelle-France parce que la nouvelle métropole était «plus capitaliste»; que si cette métropole était plus capitaliste, c'est parce qu'elle avait procédé à un «règlement hâtif de la question paysanne», c'est-à-dire l'expropriation de la petite paysannerie; que cette dépossession des petits paysans est un élément fondamental de la transition à la phase industrielle du capitalisme; que la «rupture structurelle» fut le résultat de l'exportation par l'Angleterre de cet «élément structurel» dans la vallée du Saint-Laurent par l'intermédiaire de marchands-seigneurs qui mirent en oeuvre, quoique inconsciemment, le processus de «rabattement» du capitalisme sur le féodalisme en créant les conditions de dépossession massive des paysans canadiens-français. Cette thèse soulève la question fondamentale suivante: compte tenu de ce qui a été précédemment exposé, comment la nouvelle métropole britannique aurait-elle pu, à partir de 1760 , «exporter» un phénomène socio-économique qui ne s'est jamais produit chez elle au 18e siècle, pas même au siècle suivant? Et faut-il alors s'étonner de ne trouver aucun indice vraiment significatif d'un tel mouvement dans le BasCanada de la fin du $18 \mathrm{e}$ siècle et de la première moitié du $19 \mathrm{e}$ ?

\section{$B$ - La révolution industrielle en Angleterre}

Gérald Bernier a écrit que l'on peut situer «les débuts de la révolution industrielle en Angleterre à peu près à l'époque où survient la Conquête» et que «toute entreprise coloniale tend à reproduire une partie des structures métropolitaines» ${ }^{48}$. La rupture serait immédiate après 1760 puisque, on l'a vu, sous l'effet de la métropole britannique, le procès d'articulation des modes de production est inversé en faveur du mode de production capitaliste et les activités des marchands-seigneurs

\footnotetext{
46 François Crouzet, «Agriculture et révolution industrielle...», op. cit., 73. E. P. Thompson, op. cit., 470-472.

47 Louis Bergeron, «La «révolution agricole» en Angleterre», op. cit., 232

48 Gérald Bernier, «La rupture structurelle...», op. cit., 71, note 4.
} 
britanniques créent en partie les conditions d'émergence de la phase industrielle. Pour sa part, Ta Van insiste sur l'avance prise par l'Angleterre en raison des révolutions de 1642-1660 et 1688 qui auraient consacré comme classe hégémonique «une fraction capitaliste foncière» et marchande porteuse de «l'avenir du capitalisme anglais» ${ }^{49}$.

Mais quelle était la situation réelle de l'industrie anglaise au milieu du 18e siècle? Quel fut le processus de la révolution industrielle dans ce pays au cours de cette période? En somme, qu'est-ce que l'Angleterre aurait pu «exporter» entre 1760 et 1800 qui pourrait justifier l'idée d'une rupture structurelle immédiate?

Depuis plus d'un demi-siècle, des débats sur la révolution industrielle en Angleterre opposent les historiens de l'économie. On ne saurait, par conséquent, régler dans cet article une question aussi complexe. Il est cependant possible de préciser certains points essentiels. Ainsi, W. A. Cole ${ }^{50}$ a défini la révolution industrielle en Angleterre comme l'émergence d'une croissance économique de type moderne, c'est-à-dire sans interruption brutale de crises cycliques d'Ancien Régime. Dans un premier temps, l'amélioration de l'économie à partir des années 1760 serait liée à une généralisation des innovations technologiques ${ }^{51}$, imputable à une demande accrue que les méthodes traditionnelles de production ne pouvaient satisfaire. Le gonflement du marché interne, consécutif à la croissance démographique, a forcé les pionniers de l'industrie moderne à produire plus et à un coût moindre en appliquant de nouvelles techniques. Eric Hobsbawm ${ }^{52}$ a aussi vu un facteur essentiel de développement dans l'expansion d'un marché extérieur de moindre dimension que le marché interne, mais au rythme de croissance plus rapide. Enfin, à l'instar de John U. Nef ${ }^{53}$, il faut aussi considérer des phénomènes qui annoncent dès le $16 \mathrm{e}$ siècle et le début $\mathrm{du} 17 \mathrm{e}$, les changements de la fin du $18 \mathrm{e}$.

Il est en effet indéniable que l'économie anglaise progressa au cours de ce siècle. Dans l'agriculture, l'influence du modèle «hollandais» (culture intensive, rotations accélérées, recul de la jachère) permit une meilleure productivité. Dans l'industrie, certaines techniques nou-

\footnotetext{
$49 \quad$ Ta Van, op. cit., 19.

50 W. A. Cole, «18th Century Economic Growth Revisited», Explorations in Economic History, 4 (1973): 327-348.

David Landes a montré que la généralisation des innovations technologiques au 18e siècle fut déterminante dans le cas anglais: David Landes, «Technological Change and Development in Western Europe, 1750-1914», in H. J. Habakkuk et M. Postan, dir., The Cambridge Economic History of Europe, vol. 6: The Industrial Revolution and After (Cambridge, Cambridge University Press, 1965), 274-454.

52 Eric Hobsbawm, L'ère des révolutions (Paris, Fayard, 1969), 49-50.

53 Pour un résumé des travaux de John U. Nef, voir François Crouzet, «Angleterre et France au XVIIIe siècle. Essai d'analyse comparée de deux croissances économiques», AESC, 21,2 (mars-avril 1966): 254-255, et Fernand Braudel, Civilisation matérielle, économie et capitalisme. Le temps du monde (Paris, Armand Colin, 1979), tome 3: 477-480.
} 
velles et une plus grande utilisation du charbon créèrent un "climat» favorable aux innovations qui fut d'une grande importance au 18e siècle. Dans le domaine financier, la création de la Banque d'Angleterre (1694) allait donner à ce pays certains avantages, notamment par rapport à la France qui échoua à appliquer ces nouvelles méthodes avec la faillite de Law $(1720)^{54}$. Ces améliorations furent cependant réalisées sans bouleversement de l'équilibre économique traditionnel.

Après une première moitié du $18 \mathrm{e}$ siècle moins brillante que les années $1600-1650$, il fallut attendre les années $1760-1820$ pour que la combinaison de plusieurs facteurs rende possible la révolution industrielle. Les effets conjugués de la hausse démographique, du gonflement consécutif du marché interne, de l'expansion du marché colonial, de l'augmentation de l'offre d'emplois résultant de la modernisation de l'agriculture et de l'application de nouvelles techniques dans le secteur manufacturier naissant, elles-mêmes déterminées par une pression accrue de la demande, créèrent les conditions d'émergence de transformations économiques fondamentales sans pour autant modifier de façon substantielle, entre 1760 et 1820 , les taux annuels de croissance du revenu national et de la formation de capital par rapport à ce qu'ils étaient entre 1700 et $1760^{55}$. C'est pourquoi il est important de distinguer la première phase de la révolution industrielle, celle de 1760 à 1820, de la deuxième, de 1820 à 1850, qui fut beaucoup plus spectaculaire et qui se traduisit par des taux de croissance supérieurs et continus.

Au cours de cette première phase, le leading sector fut l'industrie du coton. Pourquoi cette industrie plutôt qu'une autre? Parce qu'au début de la révolution industrielle, il a fallu, selon Peter Mathias ${ }^{56}$, une industrie liée à un marché expansible, dont la mécanisation d'un secteur, réalisable sans investissements considérables, puisse entraîner la mécanisation des autres étapes de fabrication et, à plus long terme, transformer complètement le procès de production. Stimulée par la demande d'une vaste clientèle, notamment celle des colonies, à la recherche de tissus légers, l'industrie du coton a été la première à

\footnotetext{
54 François Crouzet, «Angleterre et France au XVIIIe siècle...», op. cit., 259.

55 Jeffrey G. Williamson, «Why Was British Growth so Slow During the Industrial Revolution?», The Journal of Economic History, 44,3 (September 1984): 687, 689, et, Charles Feinstein, «Capital Formation in Great Britain», in Peter Mathias et Michael Postan, dir., The Cambridge Economic History of Europe, volume 7: The Industrial Economics Capital, Labour and Enterprise (Cambridge, Cambridge University Press, 1978), 28-96. Le taux annuel de croissance per capita du «revenu national» aurait été de $0,33 \%$ entre 1770 et 1815 contre $0,86 \%$ entre 1815 et 1841 et $1,5 \%$ après 1850 . Quant à la part du revenu national consacrée à l'investissement intérieur, elle passe de $9 \%$ dans les années 1760 à 14\% dans les années 1850 . Jusqu'à tout récemment, on croyait, à l'instar de Phyllis Deane et W. Cole, que les taux de croissance avaient été à partir des années 1780 très supérieurs. Mais ces travaux sont aujourd'hui dépassés. Pour une critique détaillée de Deane et Cole, voir F. R. Crafts, «British Economic Growth: Review of the Evidence», The Economic History Review, 36,3 (May 1983): 177-199.

56 Peter Mathias, The First Industrial Nation. An Economic History of Britain: 1700-1914 (Londres, Methuen, 1969), 129-133.
} 
répondre à ces conditions. Pour Fernand Braudel, «la révolution du coton se distingue des révolutions antérieures par un fait crucial: elle a réussi; elle n'a pas sombré dans un retour à la stagnation de l'économie; elle inaugure une croissance longue qui finira par être une «croissance continue» ${ }^{57}$. Lors de l'étape initiale de la révolution industrielle, soit de 1760 à 1820, seules les opérations de filage dans l'industrie du coton furent mécanisées. La période charnière, celle des transformations irréversibles qui allaient faire basculer l'Angleterre dans le monde massivement industriel, fut celle des années 1820-1850. La mécanisation des opérations de tissage dans l'industrie cotonnière fut complétée uniquement au cours de ces trente années. Quant à l'industrie lainière, longtemps en retrait, son retard fut comblé entre 1835 et 1850 , période au cours de laquelle le nombre des métiers mécaniques dans cette industrie passa de 3000 à 33000 , provoquant la ruine dramatique d'artisans encore très puissants au tout début du 19e siècle. Dans l'ensemble, l'utilisation plus systématique de la machine à vapeur et son perfectionnement, le développement du secteur minier et de la métallurgie, l'émergence spectaculaire du chemin de fer ont fait de l'Angleterre du deuxième tiers du $19 \mathrm{e}$ siècle l' $l^{\prime}$ «atelier du monde» ${ }^{58}$.

Pourtant, même en drainant à son profit, vers le milieu de ce siècle, plus du tiers des échanges mondiaux, l'Angleterre ne vivait pas encore complètement à l'heure de la grande industrie. Ainsi, en 1851, sur 1000 manufactures d'étoffes de laine, 500 comptaient moins de 10 ouvriers et 82 plus de $100^{59}$. La même année, sur les 677 plus importantes firmes métallurgiques, les deux tiers ont moins de 10 ouvriers, 34 plus de 100 et 14 plus de 350; sur 1800 usines de cotonnades, 113 seulement emploient plus de 350 ouvriers $^{60}$. En 1850, les «travailleurs de l'agriculture», malgré l'intensification du transfert de main-d'oeuvre des campagnes vers les villes, «composent encore le premier groupe professionnel du pays» et l'on estime le nombre des ouvriers salariés de la grande industrie à moins de 2 millions sur une population de 21 millions ${ }^{61}$.

On voit donc que les premières manifestations de la révolution industrielle en Angleterre, entre 1760 et 1820, bien qu'essentielles, n'ont pas totalement chamboulé ce pays. Dans ces conditions, comment, concrètement, l'Angleterre, entre 1760 et 1820 , aurait-elle pu «exporter», dans l'une de ses colonies, une nouvelle dynamique capitaliste susceptible de provoquer une «rupture» des structures économi-

\footnotetext{
57 Fernand Braudel, op. cit., 494. 3: $385-452$

François Caron, «La Grande-Bretagne vers 1815-1850», in Pierre Léon, dir., op. cit.,

59 Roland Marx, La révolution industrielle en Grande-Bretagne (Paris, Armand Colin, 1970), 231.

60 Ibid.

61 François Bédarida, op. cit., 27.
} 
ques, lorsqu'elle-même ne traverse qu'à partir de 1820 seulement et jusqu'en 1850, la phase cruciale qui la propulse dans le monde industriel? Certes, l'Angleterre de la fin du $18 \mathrm{e}$ siècle possède, par rapport à son concurrent d'outre-Manche, une certaine avance sur le plan économique, même s'il est difficile de préciser à quel degré une différence est significative entre ces deux pays ${ }^{62}$. Mais en laissant entendre que l'Angleterre des années 1760-1780 bouillonnait d'une effervescence industrielle qui s'est en fait réalisée presque un demi-siècle plus tard, on commet un anachronisme qui ne fait guère avancer l'interprétation de la Conquête. Ici, comme dans le cas de la question paysanne, il n'y a aucune preuve que les événements de 1760 aient amené une transformation majeure des structures économiques de l'ancienne NouvelleFrance. Plusieurs auteurs, pourtant rarement associés (Paquet et Wallot, Ouellet et Hamelin) ${ }^{63}$, ont soutenu que le mercantilisme est demeuré après la Conquête le cadre économique de cette colonie perdue dans la vallée du Saint-Laurent avec, comme principale base d'accumulation du capital marchand jusqu'au début du $19 \mathrm{e}$ siècle, le commerce des fourrures. Comment aurait-il pu en être autrement? Il n'y a rien dans les articles de Gérald Bernier et de Ta Van qui nous permette d'en douter.

\section{C - Les révolutions anglaises et la Révolution américaine}

Pour terminer, il est deux points sur lesquels nous voudrions faire quelques brèves remarques. D'abord, le rôle joué par l'aristocratie foncière anglaise pendant la révolution industrielle. Nous avons vu qu'outre la «dépossession de la petite paysannerie», l'autre facteur essentiel de la transition du féodalisme au capitalisme en Angleterre aurait été la

62 Pierre Léon, «L'élan industriel et commercial», in Fernand Braudel et Ernest Labrousse, dir., Histoire économique et sociale de la France (Paris, Presses universitaires de France, 1970), 2: 499-524; François Crouzet, «Angleterre et France au 18e siècle. Essai d'analyse comparée de deux croissances économiques», op . cit. Selon les calculs présentés dans ces articles, les croissances industrielle et commerciale de la France entre 1720 et 1780 étaient légèrement supérieures à celles de l'Angleterre. L'agressivité des marchands français de cette époque, particulièrement sur le continent où ils supplantèrent sur presque tous les marchés leurs concurrents anglais, la réussite de l'industrie sucrière françàise des Antilles et celle de la soie ont, entre autres, contribué au «rattrapage» de la France. Malgré cet élan, le retard contracté par la France au 17e siècle ne fut pas, selon François Crouzet, complètement rattrapé. Il faut aussi tenir compte du fait que la France du 18e siècle était un pays beaucoup plus habité (de 20 à 26 millions entre 1720 et 1780) que l'Angleterre (de 5 à 8 millions pour les mêmes années) et aux contrastes régionaux plus marqués. D'ailleurs, il est intéressant de constater qu'au départ la révolution industrielle en Angleterre a été un phénomène régional. Enfin, il est possible que la Révolution de 1789 et les guerres à partir de 1792 aient ralenti l'élan économique de la France au 18e siècle, bien que le débat sur ce sujet reste entier; voir Hubert Bonin, «La Révolution française a-t-elle bloqué la croissance économique?», L'histoire, 77 (avril 1985): 98-100.

63 Gilles Paquet et Jean-Pierre Wallot, «Sur quelques discontinuités dans l'expérience socioéconomique du Québec: une hypothèse», RHAF, 35,4 (mars 1982): 500-501. Fernand Ouellet, dans son Histoire économique et sociale du Québec 1760-1850 (Montréal, Fides, 1971), 1: 13: «L'annexion du Canada à l'empire britannique ne signifiait pourtant pas une révolution susceptible de modifier profondément et en peu de temps son rôle dans l'économie impériale. En Angleterre comme en France, le système mercantiliste était bien vivant.» 
victoire lors des révolutions du $17 \mathrm{e}$ siècle d'une «fraction capitaliste foncière et marchande porteuse de l'avenir du capitalisme anglais». Dominante aussi bien dans l'historiographie marxiste que dans l'historiographie libérale, cette proposition n'est aucunement fondée. La «fraction capitaliste foncière et marchande» n'a joué aucun rôle direct dans les changements économiques de la fin du 18e siècle.

Si l'aristocratie a participé indirectement à la révolution industrielle par la construction de routes et de canaux, dans l'ensemble, les pionniers de l'industrie moderne en Angleterre furent des gens des classes moyennes, artisans de la métallurgie, comme certains maîtres de forge, ou des yeomen devenus industriels. «Les capitaux qui permirent la création de la grande industrie vinrent en bonne partie de l'industrie elle-même.» ${ }^{64}$

Dans l'industrie textile, les aristocrates furent absents, hormis quelques exceptions que des historiens ont montées en épingle. Les premières filatures «modernes» de l'industrie cotonnière ont été mises sur pied par de petits entrepreneurs, très souvent des artisans habiles et ambitieux. Au départ, ils furent avantagés par le bas niveau de capital fixe requis soit pour l'achat ou la location de machines à filer facilement adaptables et bon marché, soit pour l'utilisation de locaux rudimentaires où l'on pouvait entasser quelques dizaines de travailleurs. Les taux de profit par contre étaient excellents: «de l'ordre de $20 \%$ du capital engagé, dans les bonnes années, 30\% chez McConnell et Kennedy, filateurs à Manchester de 1795 à $1810 .{ }^{65}$ Robert Owen a commencé de cette façon.

En ce qui concerne l'industrie du charbon, le cas des landlords du Pays de Galles montre qu'ils préféraient louer leurs mines à des entrepreneurs, surtout après 1750 . En effet, à partir de cette date, les grands propriétaires ont investi dans les enclosures et les nouvelles techniques agricoles. Pas dans l'industrie ${ }^{66}$.

Quant aux très riches négociants, les nababs des Indes orientales, les propriétaires de plantations antillaises et les «West India merchants», leurs fortunes furent surtout consacrées à l'achat de domaines fonciers dans la métropole ou en prêts hypothécaires aux planteurs. Certes, les entrepreneurs manufacturiers bénéficièrent de l'infrastructure du commerce extérieur. Mais il n'y eut pas de transfert massif du grand négoce vers l'industrie «moderne» ${ }^{67}$ au moment de la révolution industrielle.

\footnotetext{
64 François Crouzet, «La formation du capital en Grande-Bretagne pendant la révolution industrielle», in Deuxième conférence internationale d'histoire économique, Aix-en-Provence, 1962 (Paris, Mouton, 1964), 598.

65 Louis Bergeron, «La révolution industrielle...», op. cit., 331.

66 François Crouzet, «Agriculture et révolution industrielle...», op. cit., 72.

67 François Crouzet, «La formation du capital...», op. cit., 606-607.
} 
Au contraire de certaines idées reçues, le seul transfert significatif de capitaux, entre 1760 et 1820 , s'effectua de l'industrie vers la propriété foncière. Sitôt enrichis, les nouveaux industriels, comme Sir Richard Arkwright, Mathew Boulton et Simon Whitebread ${ }^{68}$ se sont empressés d'acheter des grands domaines et d'entrer dans le monde très sélect de l'aristocratie foncière.

Car il ne faudrait pas exagérer le caractère «démocratique» des révolutions du 17e siècle en Angleterre. Après 1688, la classe dirigeante anglaise demeure la haute noblesse ou «nobility», composée d'environ 300 familles: ducs de Devonshire, de Norfolk, de Bedford, de Rutland, de Newcastle; comtes de Derby, de Durham, etc. Vient ensuite la petite noblesse ou «gentry»: 3000 familles. Quelques bourgeois, banquiers, grands négociants, se grefferont à cette aristocratie. Il n'en sera pas autrement au 18e siècle, ni pendant la première moitié du $19 \mathrm{e}$ siècle $^{69}$. Dans cette Angleterre des années 1760-1850, se trouve au faîte de la pyramide sociale une classe de rentiers qui tire l'essentiel de sa richesse de l'exploitation de grands domaines fonciers confiés à des fermiers efficaces et qui profite d'institutions politiques et juridiques taillées sur mesure pour elle. Même le «Great Reform Bill» de 1832, qui fit passer le nombre des électeurs de 440000 à 717000 , laissa presque intacte la prépondérance de l'oligarchie. "The county families that sent MPs to Westminster in 1865 would have been familiar to George III in 1765, though their members wore top hats and trousers instead of cocked hats and breeches and travelled by train instead of coach.» ${ }^{70}$ De plus, les squires conserveront «en étroite alliance de fait et de droit avec les ministres de l'Église anglicane, des pouvoirs d'administration, de justice et de police, tout comme les «junkers» prussiens, jusqu'à la fin du [19e] siècle. Il faut attendre 1888 dans les villes, 1894 dans les campagnes, pour que des conseils élus remplacent les vieilles institutions nobiliaires» ${ }^{71}$.

68 Ibid., 603, 606. R. Arkwright (1732-1792). Inventeur d'une «mule jenny» perfectionnée, marchine semi-mécanique à filer le coton. Fait chevalier en 1787 . Voir André Parreaux, $L a$ société anglaise de 1760 à 1810 (Paris, Presses universitaires de France, 1966), 12-13.

69 Les chiffres provenant des évaluations de la société anglaise dans les fameuses «social tables» de Gregory King (1688), Joseph Massie (1759) et Patrick Colquhoun (1801-1803 et 1812) montrent bien cette permanence d'une aristocratie prospère. Les analyses récentes de ces chiffres, si elles modifient les estimations trop pessimistes de King sur le nombre de pauvres en 1688 , confirment le maintien de l'oligarchie. Voir Peter H. Lindert et Jeffrey Williamson, «Revising England's Social Tables 1688-1812», Explorations in Economic History, 19,4 (October 1982): $385-408$

70 Norman Gash, Aristocracy and People, Britain 1815-1865 (Londres, Arnold, coll. «The New History of England», 1979), 347.

71 Yves Lequin, «La résistance des aristocraties», in Pierre Léon, dir., Histoire économique et sociale du monde (Paris, Armand Colin, 1978), tome 4: La domination du capitalisme, 314. Voir également, Arno Mayer, La persistance de l'Ancien Régime (Paris, Flammarion, 1983), 1122 et 151-163, et, W. L. Guttsman, The British Political Elite (Londres, McGibbon, 1965), 3460 . 
En somme, la révolution industrielle n'a pas détruit dans l'immédiat les pouvoirs sociaux et politiques de la noblesse anglaise. Bien sûr, les années 1820-1850 furent marquées par l'émergence de phénomènes comme la pénétration du marché, la stabilisation de la monnaie, l'adoption du credo libre-échangiste et du laisser-faire tempérés par des interventions pragmatiques qui firent de l'Angleterre le moule de l'ordre économique libéral, avant même que le triomphe de la grande industrie ne soit complet ${ }^{72}$. Il est incontestable que la bourgeoisie industrielle marqua des points importants lorsqu'en 1846, le ministère Peel abrogea les «Corn Laws», répondant ainsi aux voeux de l'«Anti-Corn-LawLeague» de Richard Cobden. Mais une partie de l'aristocratie, à l'instar du comte Russell (père du «Reform Bill» de 1832) et de Sir Robert Peel, avait très bien compris que le maintien de ses prérogatives était lié à une subtile adaptation au changement. Et il a fallu la crise agricole des années 1875-1895 pour assister à un net affaiblissement économique de l'aristocratie et, du coup, à son véritable recul social et politique. Symboles de ce phénomène, Lloyd George fut, en 1905, le premier homme du peuple à siéger au gouvernement, alors que Herbert $\mathrm{H}$. Asquith devint, en 1908, le premier politicien non-noble à être élu premier ministre sans avoir été coopté par la noblesse, comme le fut Disraéli.

L'idée selon laquelle l'Angleterre de Tom Jones et de Barry Lindon serait issue des révolutions «bourgeoises» du $17 \mathrm{e}$ siècle n'est donc pas recevable. Pas plus, d'ailleurs, que les explications de Gérald Bernier et de Ta Van sur le comportement des marchands britanniques qui s'installèrent à Montréal et à Québec après 1760 . Loin de répondre à une logique capitaliste souterraine conduisant à «l'expropriation de la paysannerie», leur empressement à acquérir des seigneuries correspondait plus vraisemblablement à la logique d'une société métropolitaine dominée par la noblesse.

Ce dernier aspect avait pourtant été mis à jour par Pierre Tousignant ${ }^{73}$. Et, récemment, Jean-Marie Fecteau a bien montré que le modèle de régulation sociale installé par l'Angleterre au Canada après la Conquête n'était pas celui d'une société bourgeoise ${ }^{74}$.

Par ailleurs, il est curieux que l'on puisse disserter ad nauseam des relations économiques entre l'Angleterre et la «Old Province of Quebec» à la charnière du $18 \mathrm{e}$ et du $19 \mathrm{e}$ siècles sans mentionner une seule fois l'existence des Treize colonies américaines. Pourtant, s'il y eut après la Conquête un événement susceptible de provoquer un change-

\footnotetext{
72 Karl Polanyi, The Great Transformation (Boston, Beacon Press, 1957), 320 p.

73 Pierre Tousignant, «Problématique pour une nouvelle approche de la Constitution de 1791», RHAF, 27,2 (septembre 1973): 181-234.

74 Jean-Marie Fecteau, «Régulation sociale et répression de la déviance au Bas-Canada au tournant du 19e siècle (1791-1815)», RHAF, 38,4 (printemps 1985): 499-521.
} 
ment radical de l'économie, ce fut bien la Guerre d'Indépendance américaine.

Dans la lutte qui opposa, très rapidement après leur arrivée au Canada, les «Montrealers» aux Bostoniens, les premiers poursuivaient un objectif fondamental: faire du fleuve, au détriment de la façade atlantique, le centre commercial de l'Amérique du Nord. Les Todd, Frobisher, Henry, Peter Pond, Paterson, Grant, McTavish, McGill, etc. ont rêvé d'un tel empire commercial ${ }^{75}$. La Révolution américaine aurait dû être l'occasion unique de distancer les concurrents de la Nouvelle-Angleterre. Il n'en fut rien. Au contraire. Par la signature du traité de Versailles (1783), les négociateurs britanniques, en retour d'une liberté commerciale entre les deux pays très avantageuse pour l'Angleterre, consentirent aux Américains le tracé d'une frontière qui leur concédait tout le bassin sud des Grands Lacs qu'ils n'avaient pourtant jamais conquis militairement. L'unité géographique du commerce des fourrures, qui avait Montréal comme point de départ, était ainsi détruite. Le traité de Jay (1794), en confirmant les frontières de 1783, brisa les espoirs des marchands montréalais. Ainsi, la Grande-Bretagne, en fonction d'une logique certes impérialiste, mais aux rouages différents de ceux décrits par Gérald Bernier et $\mathrm{Ta} \mathrm{Van}^{76}$, porta des coups très préjudiciables aux intérêts économiques canadiens.

En s'assurant de liens d'échanges commerciaux avec les ÉtatsUnis, les diplomates britanniques protégeaient le marché extérieur le plus important de leur pays. Les États-Unis absorbaient 37\% des produits fabriqués et exportés par l'Angleterre en 1772, 57\% en $1797^{77}$. Avec leurs 3734000 habitants en 1790, les Américains furent les principaux clients des Anglais et ils le restèrent encore un certain temps, tout en fournissant des matières premières aussi indispensables que le coton. On comprend alors que les réclamations des «Montrealers» ne furent pas entendues, ni en 1783, ni en 1794, ni en 1815. De même, un demi-siècle plus tard, lorsque le ministère Peel, malgré la vive opposition d'une partie de l'aristocratie, adopta le libre-échange à partir de 1846 , créant ainsi une véritable rupture structurelle, les descendants de la première génération de marchands montréalais anglophones n'eurent pas plus de succès, les problèmes de la colonie étant de peu de poids face aux intérêts économiques supérieurs de la Grande-Bretagne. La suite est bien connue: manifeste annexionniste (1849), réciprocité avec

\footnotetext{
75 Donald Creighton, The Empire of the St-Lawrence (Toronto, MacMillan, 1956), $440 \mathrm{p}$. Gérald Bernier et Ta Van montrent que la Grande-Bretagne «plus capitaliste» a suscité l'avènement de la «phase commerciale» et freiné l'émergence de la «phase industrielle». Mais, concrètement, les intérêts diplomatiques et économiques de la Grande-Bretagne l'ont conduit à réduire l'élan de la «phase commerciale» telle qu'envisagée par les marchands montréalais.

P. J. Cain et A. G. Hopkins, «The Political Economy of British Expansion Overseas, 1750-1914», Economic History Review, 33,4 (1282 November 1980): 463-490.
} 
les Américains (1854), refus américain de reconduire ce traité de réciprocité, Confédération.

\section{CONCLUSION}

En se référant aux philosophes de l'histoire du début du 20e siècle, René Rémond eut ce commentaire:

Des mauvaises langues insinueraient que les philosophes de l'histoire sont d'autant plus libres d'échafauder leurs synthèses et de proposer des interprétations synthétiques et unitaires de l'évolution historique qu'ils ne sont pas le moins du monde embarrassés par une connaissance de l'histoire sur laquelle ils exercent leur activité spéculative. A eux les larges horizons, les synthèses audacieuses, les raccourcis saisissants. ${ }^{78}$

Méfiants à l'endroit de ces «raccourcis saisissants», les historiens ont peut-être trop tendance à verser dans l'excès contraire. Or, «pas de théorie, pas d'histoire», professait Werner Sombart. Mais nous avons vu dans quel obscurantisme nous plongent les révélations des «lois de l'histoire» qui se manifestent par des phénomènes imaginaires et des anachronismes.

Tel que posé dans les articles que nous avons étudiés, le problème de la transition du féodalisme au capitalisme apparaît comme une comparaison de gros sabots qui ne laisse guère de place à l'appréhension de dynamiques économiques, sociales et politiques très complexes. Ce qui n'est pas du «féodalisme» fait partie intégrante du seul «vrai» capitalisme, celui de la «grande industrie», et ce qui n'est pas du capitalisme industriel est «féodal», ce mot étant manifestement entendu chez ces auteurs au sens d'une «grande noirceur» politique et sociale. De plus, selon la théorie, le passage d'un mode de production à un autre s'expliquerait par le fait qu'à un certain moment, la poussée des forces productives est telle qu'elle conduit à l'éclatement des rapports sociaux de production et, surtout, à l'émergence d'une nouvelle classe dominante correspondant au nouveau mode de production. Mais en Angleterre, entre 1780 et 1850 , des changements économiques fondamentaux se produisirent et pourtant l'aristocratie resta puissante jusqu'à la fin du $19 \mathrm{e}$ siècle. Comment alors rendre compte théoriquement de cette Angleterre du 19e siècle? Car même si l'Angleterre des années 17801850 est toujours dominée par une classe qui tire sa richesse de l'exploitation du travail de la terre, même si la grande industrie, malgré les mutations économiques de cette période, n'y est pas encore tout à fait la norme généralisée, cette Angleterre est-elle pour autant «féodale»? Et les activités de John Richardson, grand bourgeois montréalais, l'un

78 René Rémond, cité dans, Maurice Lagueux, Cours de philosophie de l'histoire, Université de Montréal, 1967, texte polycopié, 6. 
des fondateurs de la Banque de Montréal (1817) et des promoteurs du canal de Lachine (1824), sans relever du capitalisme de la grande industrie, sont-elles nécessairement «féodales»? En fait, ce que l'on qualifie lapidairement de "phase transitoire» pourrait recouvrir des particularités essentielles qu'une approche théorique fondée sur une succession simpliste de modes de production ne peut saisir.

A remarquer également les imprécisions au coeur même de l'argumentation. On nous apprend en effet que l'histoire du Canada est marquée entre 1760 et 1850 d'un «double procès de transition»: celui du féodalisme au capitalisme et à l'intérieur de ce dernier, celui de la «phase marchande» à la "phase industrielle». Mais plus loin, il est affirmé que le capitalisme marchand relève toujours du féodalisme et que le «vrai» capitalisme apparaitt avec la grande industrie. Que signifie alors exactement cette notion de «transition», l'apparition du «vrai» capitalisme ne pouvant être que soudaine puisque tout ce qui la précède est défini comme «féodal»?

Dans sa rigoureuse critique des théories de Marx sur la révolution agricole en Angleterre, Kins Collins a écrit: «Proof of the falsity of the theory is not based on Marx's having used false statements to construct it, but rather solely on the fact that after the theory was constructed, false statements were derived from it. ${ }^{79}$ Voilà sans doute le plus grave danger des contributions de certains politicologues.

Enfin, si la Conquête n'a pas provoqué de rupture immédiate des structures économiques, elle reste l'événement à partir duquel prend racine la question nationale. Un modèle théorique devrait donc être construit de façon à intégrer une telle spécificité et l'interaction des différentes dimensions d'une société. En somme, si le questionnement de l'ensemble est indispensable, il doit éviter les explications trop centrées sur un seul facteur, se méfier de l'application mécanique d'une vision téléologique de l'histoire et tenir compte des travaux des historiens.

79 Kins Collins, op. cit., 368. 